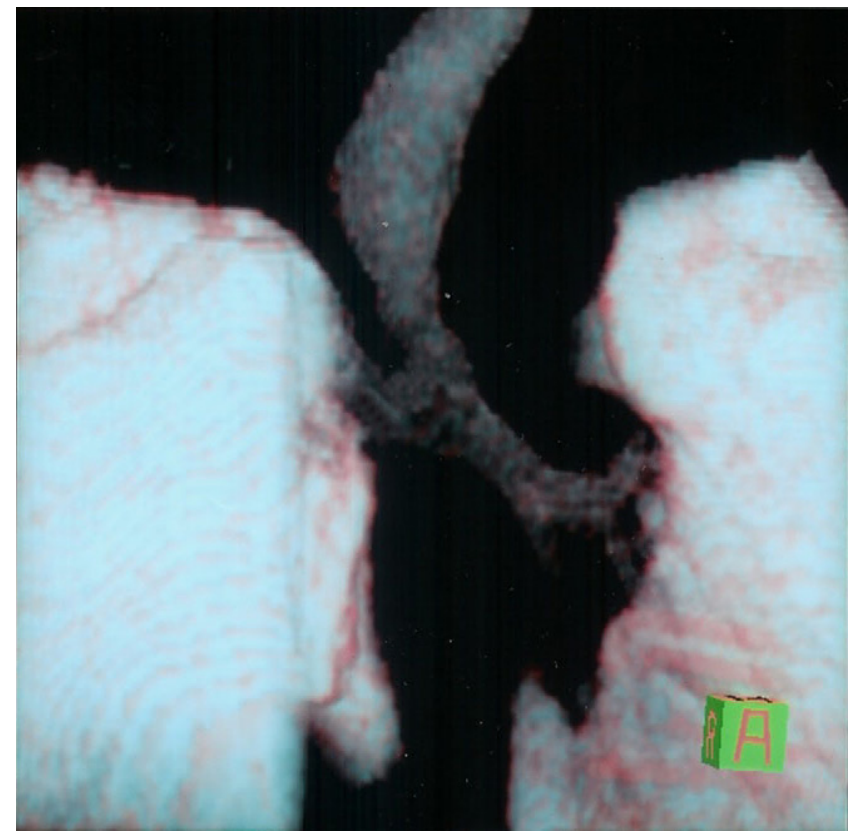

Figure 2. Six months after Barclay's procedure using the continuous suture technique. (Siemens LSO HI-REZ Biograph 16; Siemens Medical Systems, Munich, Germany). using 4-0 polypropylene (Figure 1). Subsequently, the anterior portion is sutured, and the anastomosis is completed without any difficulty. No additional precautions are taken to provide ventilation during the procedure. Apnea after complete saturation generally facilitates completion of the anastomosis.

We have performed end-to-side bronchial anastomoses in a few cases in which the continuous technique was used only for the posterior part of the anastomosis. In the reported case, end-to-side anastomosis was performed using the continuous technique for the entire circumference, and it resulted in a satisfactory outcome. This suggests that continuous suturing seems to be an adequate technique even for end-to-side bronchial anastomoses.

\section{References}

1. Kutlu CA, Goldstraw P. Tracheobronchial sleeve resection with the use of a continuous anastomosis: results of one hundred consecutive cases. J Thorac Cardiovasc Surg. 1999;117:1112-7.

2. Barclay RS, McSwan N, Welsh TM. Tracheal resection without the use of grafts. Thorax. 1957;12:177-80.

3. Mitchell JD, Mathisen DJ, Wright CD, Wain JC, Donhue DM, Allan JS, et al. Resection for bronchogenic carcinoma involving the carina: longterm results and effect of nodal status on outcome. J Thorac Cardiovasc Surg. 2001;121:465-71.

4. Mitchell JD, Mathisen DJ, Wright CD, Wain JC, Donhue DM, Moncure AC, et al. Clinical experience with carinal resection. J Thorac Cardiovasc Surg. 1999;117:39-53.

\title{
Thrombus in the left superior pulmonary vein after left upper pulmonary lobectomy
}

Eiki Nagaoka, MD, Makoto Yano, PhD, Takahiko Sugano, MD, and Takamichi Miyamoto, MD, Tokyo, Japan

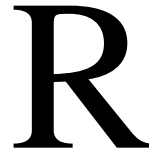

enal infarction related to lung resection is rarely reported. We first encountered a case of renal thromboembolism midterm after lung resection. Creating a stump of pulmonary vein after major lung resection is among the

From the Department of General Thoracic Surgery, Musashino Red Cross Hospital, Musashino, Japan.

Received for publication Oct 4, 2007; accepted for publication Nov 21, 2007.

Address for reprints: Eiki Nagaoka, MD, General Thoracic Surgery, Musashino Red Cross Hospital, 1-26-1 Kyonancho, Musashino, Tokyo, Japan 180-8601 (E-mail: ei.n@mm.neweb.ne.jp).

J Thorac Cardiovasc Surg 2008;135:709-10

0022-5223/ $\$ 34.00$

Copyright $@ 2008$ by The American Association for Thoracic Surgery doi:10.1016/j.jtcvs.2007.11.035 few ways in which surgeons can generate a thrombus in the arterial system, which may lead to organ infarction. Infarction after lung resection, however, has rarely been reported. We present a case of renal infarction after lung resection.

\section{Clinical Summary}

A 76-year-old man underwent resection of the left upper lobe for lung cancer. Thirteen months after the operation, he sought treatment with severe right flank pain. A computed tomographic scan of the chest and abdomen, and of the pelvis with intravenous injection of contrast medium, revealed a large, wedge-shaped infarction of the left kidney (Figure 1). We detected a ball thrombus floating in the stump of the left superior pulmonary vein (LSPV), which we had left at the operation 13 months before. We consider this infarction of the kidney to be caused by thromboembolism.

We admitted the patient and started anticoagulation therapy with heparin (20,000 units/d intravenously) and warfarin sodium (3 mg/ d). Twelve days after the anticoagulation therapy had been started, 

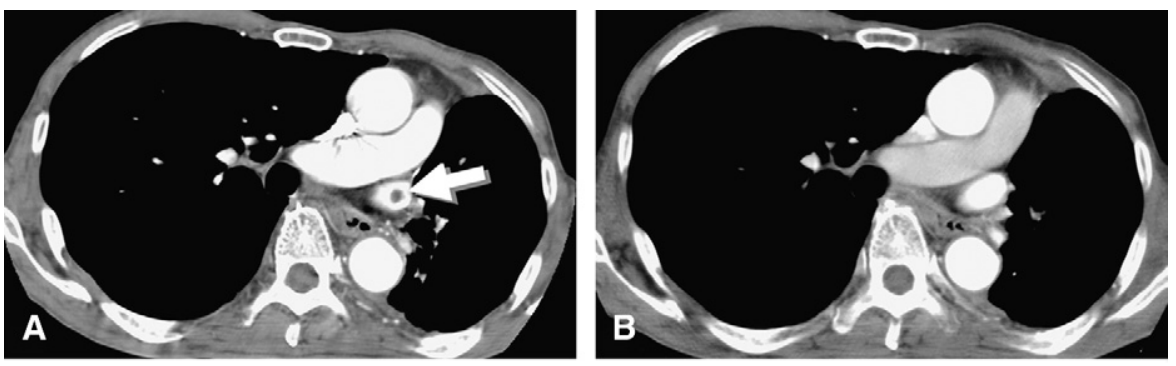

Figure 1. Computed tomographic scans of chest and abdomen. A, Round defect of contrast media in left superior pulmonary vein (arrow). B, Eleven months later, thrombus had disappeared. C, Large wedge-shaped defect in left kidney. D, One month after renal infarction, atrophy of left kidney was seen.
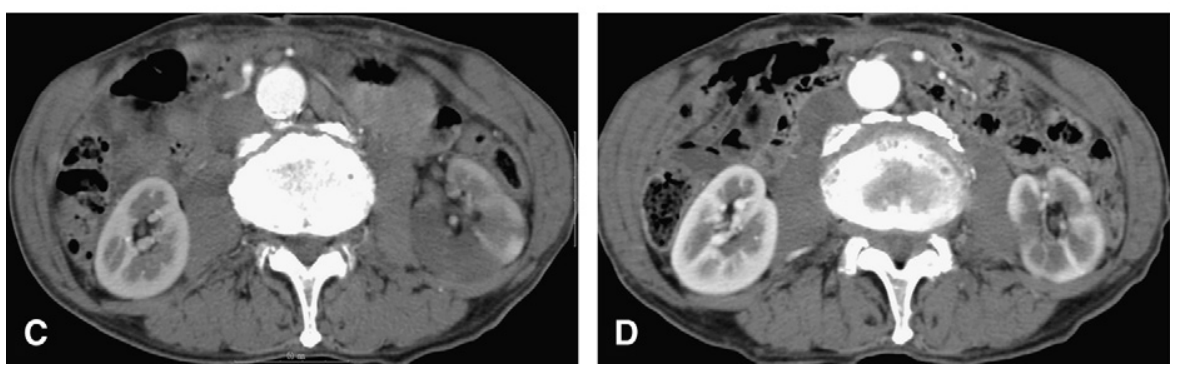

the thrombus in the LSPV seemed to be smaller on transesophageal echocardiography than it had been on the day of admission. We stopped the heparin treatment, and with $2.5 \mathrm{mg}$ warfarin per day kept the prothrombin international normalized ratio at about 2.0. Seventeen days after admission, the patient was discharged. In continuing follow-up, no new infarction has been observed.

\section{Discussion}

Abdominal organ infarction after lung surgery is rare, but it may cause serious diseases, such as renal failure, intestinal ischemia, or splenic infarction. We often ligate the stumps twice to avoid bleeding. Surgeons are not usually concerned with the size of the stumps, however, and without such concern, a thrombus may form in the stump (a blind-ended vessel) and ultimately embolize one or more organs.

There have been three case reports of abdominal organ infarction after lung surgery ${ }^{1,2}$ and one report of lower extremity thrombo-

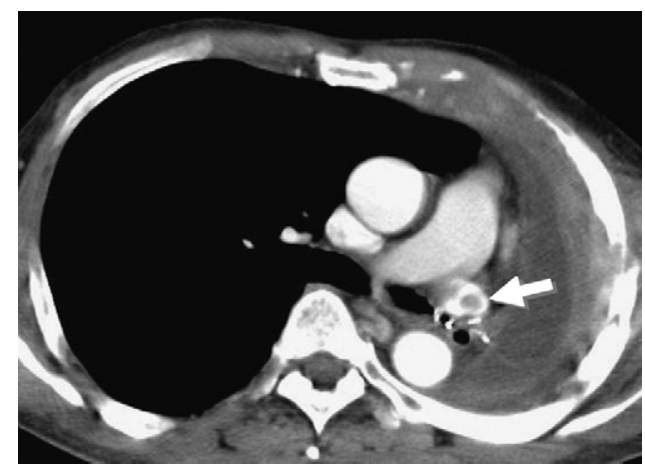

Figure 2. Computed tomographic scan of chest from another patient who had thrombus in left superior pulmonary vein (arrow). embolism. ${ }^{3}$ In the reports of abdominal infarction, the causes of infarction were not specified. In the case of lower extremity thromboembolism, thrombus was observed in the left atrium extending from the stump of the LSPV. All cases occurred after left upper lobectomy.

After this case, we examined patients who underwent lung surgery in our hospital. Through a series of 265 major lung resections in our hospital, 31 patients underwent contrast computed tomography for other reasons after the lung surgery. We carefully examined these computed tomographic scans and found another patient with a thrombus in the stump of the LSPV (Figure 2). We found two patients altogether, but one had dilated cardiomyopathy. His cardiac function was greatly reduced. For this reason, we excluded that patient.

The LSPV tends to be left longer than other pulmonary veins for anatomic reasons. This may have been the cause of thrombosis in both our cases, as both thrombi were in the LSPV.

We warn that although the occurrence is very low, thrombus may be formed in the LSPV, and some organs may be infarcted. It is therefore necessary to ligate the pulmonary vein as short as possible.

\section{References}

1. Oura H, Hirose M, Aikawa H. Abdominal organ infarction encountered immediately after surgery of primary lung cancer. Kyobu Geka. 2005; 58:137-42.

2. Borrego Hernando J, Fernández Fernández E, Galbis San Juan F. Escudero Barrilero A. [Renal infarction following pulmonary surgery in a 13-year-old boy.] [Article in Spanish]. Arch Esp Urol. 1994;47: 1019-21.

3. Seki M, Endo M, Kidani M, Kobayashi H, Sato H, Noto T. [A rare case of left atrial thrombus after left upper pulmonary lobectomy.] [Article in Japanese]. Nippon Kyobu Geka Gakkai Zasshi. 1989;37:1371-5. 